\title{
Intoxicación por munición de plomo en aves silvestres en España y medidas para reducir el riesgo
}

\author{
R. Mateo ${ }^{1, *}$, N. Vallverdú-Coll1 ${ }^{1}$, M.E. Ortiz-Santaliestra ${ }^{1}$ \\ (1) Instituto de Investigación en Recursos Cinegéticos (IREC), CSIC-UCLM-JCCM, Ronda de Toledo s/n, 13071 Ciudad Real, España \\ * Autor de correspondencia: R. Mateo [rafael.mateo@uclm.es]
}

> Recibido el 10 de febrero de 2013, aceptado el 26 de mayo de 2013.

\begin{abstract}
Mateo, R. Vallverdú-Coll, N., Ortiz-Santaliestra, M.E. (2013). Intoxicación por munición de plomo en aves silvestres en España y medidas para reducir el riesgo. Ecosistemas 22(2):61-67. Doi.: 10.7818/ECOS.2013.22-2.10

La intoxicación por ingestión de munición de plomo en aves silvestres es conocida en España desde finales del siglo pasado. Elevadas densidades de perdigones de plomo en diversos humedales de importancia internacional producen tasas de ingestión de perdigones cercanas al 70 \% en especies como el ánade rabudo (Anas acuta) y el plumbismo llega a ser una de las principales causas de mortalidad de la amenazada malvasía cabeciblanca (Oxyura leucocephala). El plumbismo también ha sido descrito en diversas especies de aves rapaces y se ha comprobado que la exposición al plomo es elevada en las especies más carroñeras y oportunistas. La contaminación de la carne de caza también ha sido objeto de estudio, debido a que en España se da la particularidad de cocinarla en escabeche, lo que incrementa la transferencia de plomo de la munición a la carne. Por el momento, la única acción llevada a cabo para reducir el riesgo de exposición al plomo de la munición en aves y humanos ha sido la prohibición desde 2001 del uso de perdigones de plomo en humedales protegidos. En esta revisión se hace un repaso a los estudios llevados a cabo en España sobre la contaminación por el plomo de la munición y el efecto que están teniendo las medidas hasta el momento adoptadas para reducir este riesgo.
\end{abstract}

Palabras clave: caza, contaminación, metales pesados, plumbismo

Mateo, R. Vallverdú-Coll, N., Ortiz-Santaliestra, M.E. (2013). Poisoning by ingestion of lead ammunition in wild birds in Spain and measures taken to reduce this risk. Ecosistemas 22(2):61-67. Doi.: 10.7818/ECOS.2013.22-2.10

Poisoning by ingestion of lead ammunition in wild birds is known in Spain since the end of the last century. High densities of lead shot in various internationally important wetlands produce pellet ingestion rates close to $70 \%$ in species such as the northern pintail (Anas acuta) and lead poisoning becomes a major cause of mortality of the endangered white-headed duck (Oxyura leucocephala). Lead poisoning has also been described in several species of birds of prey, mainly scavengers. The contamination of game meat has also been studied, because in Spain it is usually cooked with vinegar, increasing the transfer of lead from ammunition to meat. For now, the only action taken to reduce the risk of exposure to lead from ammunition in birds and humans has been the banning since 2001 of lead shot in protected wetlands. Here, we review the studies carried out in Spain on lead contamination caused by the ammunition and the effect of the measures taken to reduce this risk.

Keywords: contamination, heavy metals, hunting, plumbism

\section{Introducción}

Las aves silvestres pueden resultar expuestas al plomo a través de diversas vías y a partir de diferentes fuentes de dicho metal pesado. En el entorno de zonas afectadas por la contaminación originada por la minería o las fundiciones se puede dar la exposición por vía inhalatoria o a través de la ingestión de suelo o alimentos contaminados (Mateo et al. 2006). Sin embargo, la forma de exposición más frecuentemente asociada con una intoxicación clínica por plomo es la ingestión de munición de plomo utilizada para la caza (Guitart et al. 1999). Las aves silvestres ingieren munición de plomo por dos motivos. Las especies con un estómago muscular más desarrollado (molleja), como son las aves acuáticas o los Galliformes, por lo general se alimentan de materias vegetales duras (semillas) o animales con exoesqueleto, que hacen necesaria la ingestión regular de "grit" (gastrolitos) para romper y triturar los alimentos (Mateo et al. 2000b). Estas especies pueden ingerir los perdigones de plomo acumulados en las zonas de caza al confundirlos, principalmente, con partículas de grit (Mateo et al. 2000b, Mateo y Guitart 2000). La segunda causa de la ingestión de perdigones de plomo en aves la encontramos en las aves de presa, especialmente en las que tienen hábitos carroñeros, al alimentarse de cadáveres con munición de plomo, como perdigones o fragmentos de balas, incrustados en su carne (Cerradelo et al. 1992, Mateo et al. 1997c). Aunque en muchos casos los perdigones o fragmentos de bala son regurgitados con el material no digerido como el pelo y las plumas en forma de egagrópilas, la absorción de plomo durante la digestión de los alimentos puede ser suficiente para intoxicar a las aves de presa. El plomo absorbido produce en las aves principalmente efectos adversos en el aparato circulatorio, sistema nervioso, riñón y aparato digestivo, aunque otras funciones, como la inmune o la reproducción, también pueden verse alteradas (Mateo et al. 1998b, 2003a, Rodríguez et al. 2010). 
La presente revisión recopila los datos disponibles acerca de la intoxicación por plomo en aves silvestres en España debido a la ingestión de munición de plomo y las medidas adoptadas en la última década para reducir el riesgo de esta intoxicación.

\section{Ingestión de perdigones de plomo en aves acuáticas}

La caza de aves acuáticas se realiza en España mediante varias técnicas, dependiendo de la especie y el humedal, y es en buena medida la forma en que se practica esta caza de aves acuáticas la que determina que exista un riesgo importante de plumbismo en un humedal. Por ejemplo, la focha (Fulica atra) se suele cazar en lo que en el Delta del Ebro se conoce como "barreig", que consiste en acorralar a las aves con barcas para obligarlas a volar sobre el círculo de cazadores situados en las mismas embarcaciones o en la orilla de la laguna. Los patos son cazados principalmente desde puestos de caza situados en la orilla de lagunas o en los arrozales. La actividad de la caza en las lagunas se conoce en el Delta del Ebro como "tiradas" y se restringe a las primeras horas de la mañana. Por el contrario, la caza en los arrozales en ese mismo humedal se lleva a cabo durante las "llunes", que son las 4 noches entorno a cada luna llena de la temporada de caza. Alrededor de estos puestos de caza ("tinas") situados en los márgenes de los arrozales, los cazadores suelen cebar con grano de cereal durante toda la temporada de caza para atraer a las aves. En el pasado, en zonas como la duna del Cerro de los Ánsares de Doñana, el ánsar común (Anser anser) era cazado cuando acudía a esta duna para ingerir arena de cierto grosor para poder moler el tubérculo de la castañuela (Scirpus maritimus) en la molleja (Mateo et al. 2000a). También en Doñana, las bandadas de patos se seguían a caballo a través de los pantanos y se cazaban con armas capaces de disparar grandes cargas de perdigones. El caballo ("cabresto") servía tanto de escondite al cazador durante la aproximación como de soporte para la escopeta en el disparo (Chapman y Buck 1893).

Las mayores densidades de perdigones de plomo en estas zonas húmedas en las que se practica la caza las podemos encontrar en los lugares en que la actividad cinegética ha sido más intensa, se ha realizado desde puestos fijos y durante un largo periodo de tiempo. La alta persistencia del perdigón de plomo en el medio hace que en casi todos los humedales españoles estudiados haya podido detectarse la presencia de perdigones de plomo en sus sedimentos, con una distribución en profundidad que varía entre zonas (Guitart et al. 1994a, Bonet et al. 1995, Mateo et al. 1997b, 1998a) (Fig. 1). De modo general, las máximas densidades de perdigones en sedimento han sido detectadas en la Laguna de Medina (Cádiz), con una media de 399 perdigones $/ \mathrm{m}^{2}$ en los primeros $30 \mathrm{~cm}$ de sedimento (Mateo et al. 2007a). Puntualmente podemos encontrar densidades todavía mayores en humedales en los que hay campos de tiro deportivo como es el caso del Parque Natural de El Hondo, donde en una zona se registró una densidad de 1432 perdigones $/ \mathrm{m}^{2}$ (Bonet et al. 2004). La elevada persistencia del perdigón de plomo la hemos podido comprobar en el Parque Nacional de las Tablas de Daimiel, en el que casi 30 años después de ser prohibida la caza todavía había cerca de 100 perdigones $/ \mathrm{m}^{2}$ en los $20 \mathrm{~cm}$ superiores del sedimento (Mateo et al. 1998a). Jørgensen y Willems (1987) estimaron que la persistencia de los perdigones de plomo en el suelo podría variar entre los 30 y 300 años, dependiendo de las características y usos del suelo.

Las prevalencias de ingestión de perdigones han sido estudiadas desde finales de los años 70 mediante el examen de la molleja de aves abatidas por los cazadores (Llorente 1984, Guitart et al. 1994a, Mateo et al. 1997b, 1998a, 2000b, 2007a). Esto ha permitido observar que especies como el ánade rabudo (Anas acuta) y el porrón común (Aythya ferina) presentaban prevalencias entorno al $70 \%$. Les seguían otras especies de anátidas granívoras como el ánade azulón (Anas platyrhynchos), el pato cuchara (Anas clypeata) o la cerceta común (Anas crecca), con prevalencias cercanas al $25 \%$ (Fig. 2). Las especies que mayor tasa de ingestión de per-

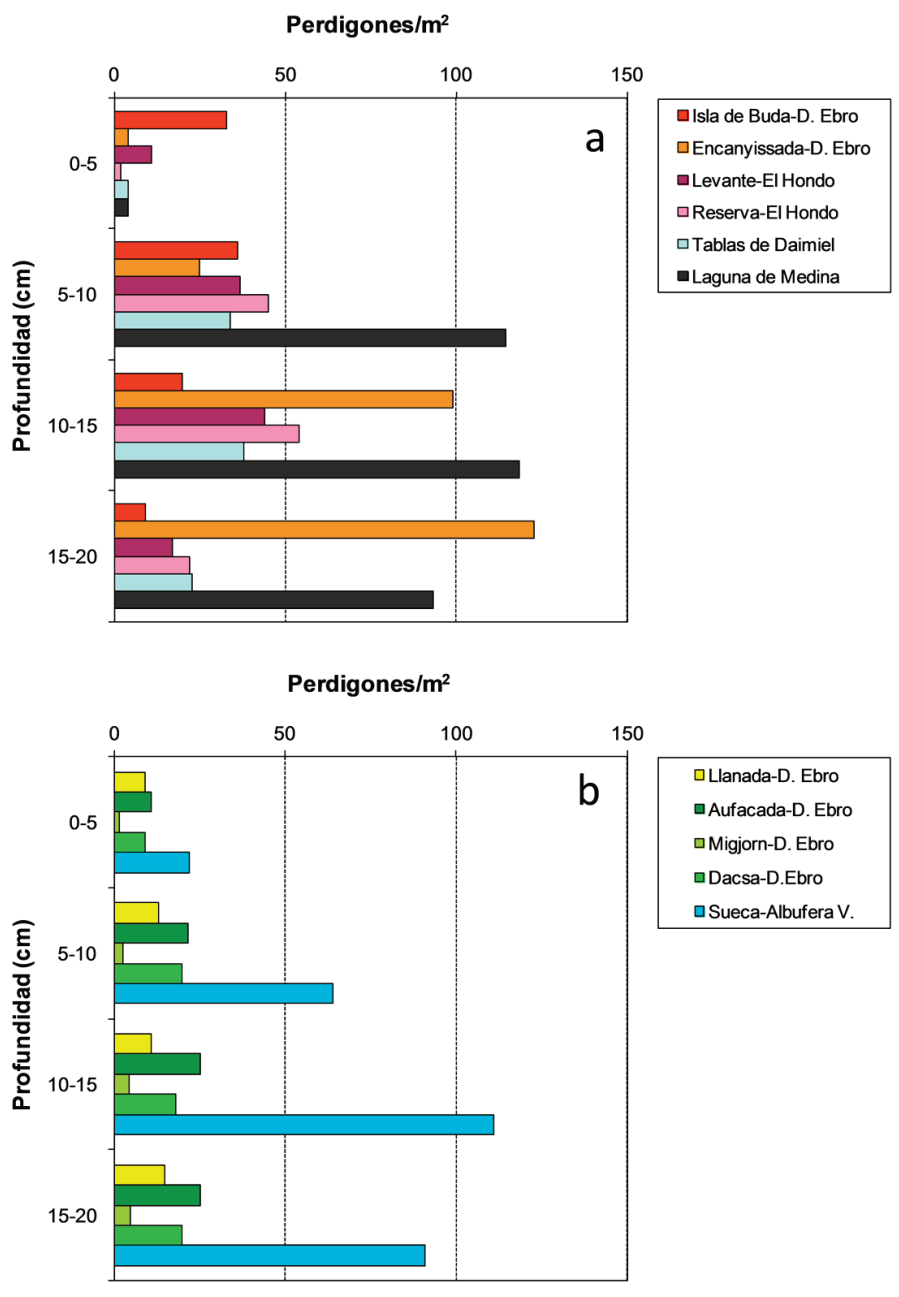

Figura 1. Densidades de perdigones de plomo en los $20 \mathrm{~cm}$ superiores de lagunas (a) y arrozales (b) de España.

\section{Prevalencia (\%)}

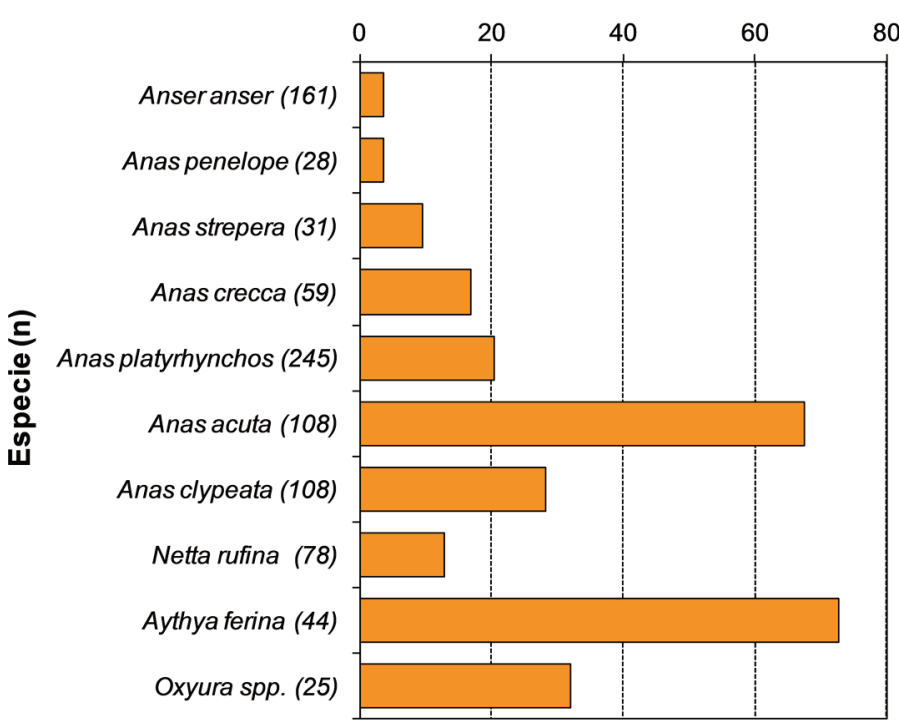

Figura 2. Prevalencia de ingestión de perdigones de plomo en anátidas de humedales españoles entre 1977 y 2004.

digones presentaban eran aquellas con dietas granívoras y que seleccionaban un tamaño de grit $>3 \mathrm{~mm}$ de diámetro (Mateo et al. 2000b). Mediante un meta-análisis de la ingestión de perdigones de plomo en 51 localidades y 27 especies de aves acuáticas, incluidos datos de España, se ha podido observar que la cantidad de 
grit en la molleja dependía de la especie, mientras que la composición en tamaños de este grit dependía más de localidad, lo que puede estar relacionado con factores como la disponibilidad de grit de diferente tamaño en el ambiente o la dieta de la especie en cada zona (Figuerola et al. 2005).

La captura de aves vivas ha permitido valorar el grado de exposición al plomo en humedales en los que la caza no está permitida actualmente. Por ejemplo, en la Laguna de Medina se ha detectado que el $19 \%$ de los ánades azulones capturados presentaban niveles de plomo en sangre $>20 \mu \mathrm{g} / \mathrm{dl}$, que son indicativos de una posible ingestión de perdigones (Martínez-Haro et al. 2011b). En los últimos años, el muestreo no invasivo de heces también ha permitido identificar la frecuencia de ingestión de perdigones de plomo en determinadas especies de aves acuáticas sin la necesidad del trampeo o de muestrear aves abatidas por cazadores (Martínez-Haro et al. 2010). La heces permiten determinar la concentración de plomo, conocer si la proporción de isótopos estables $\left({ }^{206} \mathrm{~Pb},{ }^{207} \mathrm{~Pb}\right.$ y $\left.{ }^{208} \mathrm{~Pb}\right)$ procede de la munición o de otras fuentes, valorar la cantidad de suelo ingerido mediante el análisis de cenizas insolubles en ácido o por la concentración de aluminio presente y, además, poder determinar biomarcadores que indiquen efectos adversos en las aves como son las porfirinas excretadas vía biliar y renal (Mateo et al. 2004, 2006, Martínez-Haro et al. 2011c, 2013).

La intoxicación por plomo se ha identificado como una causa importante de muerte en la malvasía cabeciblanca (Oxyura leucocephala), una especie globalmente en peligro (EN) según la UICN. Aunque la población española de esta especie ha aumentado desde menos de 100 individuos en 1978 hasta cerca de 2200 en 2007, ha sufrido un estancamiento en los últimos años y la población mundial se estima entre 7900 y 13100 aves, de las cuales unas $5300-8700$ serían individuos adultos (Torres-Esquivias 2008, BirdLife International 2012). La prevalencia de ingestión de perdigones de plomo es difícil de determinar, ya que no es una especie de caza, pero el programa español para erradicar la malvasía canela (Oxyura jamaicensis) y sus híbridos con la cabeciblanca permitieron observar una prevalencia de ingestión de perdigones del $32 \%$ en el género Oxyura. Además, el $73.3 \%$ de las Oxyura sp. encontradas muertas en los humedales españoles contenía perdigones de plomo en la molleja y el $80 \%$ tenían $>20 \mu \mathrm{g} / \mathrm{g}$ de plomo en el hígado en peso seco (p.s.) (Mateo et al. 2001b). Además, la monitorización continua de las malvasías cabeciblancas acompañada del análisis de isótopos estables de plomo confirmó la importancia de la intoxicación por plomo por la ingestión de perdigones como causa de mortalidad (Svanberg et al. 2006, Taggart et al. 2009). Otra especie de anátida en la categoría global de vulnerable (VU) como es la cerceta pardilla (Marmaronetta angustirostris) también se ve afectada por la intoxicación por plomo. El examen de aves halladas muertas debido a diferentes causas en el sur de España entre 1996 y 2001 ha permitido observar que el $32.9 \%$ de las aves habían ingerido perdigones de plomo y el $20 \%$ tenían > $20 \mu \mathrm{g} / \mathrm{g}$ p.s. de plomo en hígado (Mateo et al. 2001b, Svanberg et al. 2006).

Se estima que la mortalidad por intoxicación por plomo en aves acuáticas en Europa afectaría a cerca de un millón de animales de una población total de más de once millones de aves de 17 especies (Mateo 2009). En el Delta del Ebro, la mortalidad por intoxicación por plomo dentro de una población mayoritariamente sedentaria de ánades azulones se estimaba a principios de los años 1990 en alrededor de 9600 aves durante toda la temporada invernal (Mateo et al. 1997b). A esto se añade que las tendencias de las poblaciones invernantes de ánade rabudo y porrón común en Europa durante las últimas décadas han sido negativas (BirdLife 2004), siendo estas especies las de mayor prevalencia de ingestión perdigones de plomo en el sur de Europa a finales del siglo XX (Mateo 2009). Si tenemos en cuenta todas las especies de anátidas cazadas en Europa, se observa una relación significativa entre la tendencia del conjunto de poblaciones invernantes y la prevalencia total de ingestión de perdigones de plomo en cada especie (Mateo 2009).

Además de los Anseriformes, la ingestión de perdigones y la consiguiente intoxicación por plomo ha sido observada en otros grupos de aves acuáticas como Ciconiformes, Phoenicopteriformes, Ralliformes y Charadriformes. En España se han producido varias mortalidades masivas por plumbismo en flamenco común (Phoenicopterus ruber), como fue el caso de 22 flamencos comunes encontrados muertos en 1991 en Doñana (Ramo et al. 1992) y otros 106 individuos muertos entre 1992 y 1994 en El Hondo y las Salinas de Santa Pola (Mateo et al. 1997a). Además, el $24 \%$ de los flamencos comunes cazados de forma ilegal en el Delta del Ebro presentaban perdigones de plomo ingeridos en la molleja (Mateo 1998). En el calamón común (Porphyrio porphyrio), se observaron perdigones de plomo en la molleja en el $7.4 \%$ de las aves muestreadas en Doñana hace cuatro décadas (Rodríguez e Hiraldo 1975), pero en un estudio más reciente no se llegó a observar la ingestión de perdigones en aves trampeadas y tan solo un individuo encontrado muerto presentaba perdigones en su molleja (Mateo et al. 2007a). La intoxicación por plomo ha sido diagnosticada en base a la determinación de plomo en sangre e hígado en morito (Plegadis falcinellus) (R. Mateo, dato inédito), aguja colinegra (Limosa limosa) (Mateo 1998), avefría (Vanellus vanellus) y avoceta (Recurvirostra avosetta) en el Delta del Ebro (Guitart et al. 1994a, 1994b).

\section{Intoxicación por plomo en galliformes}

La densidad de perdigones de plomo en los hábitats terrestres en los que se ha cazado ha sido en general muy poco estudiada. Únicamente Ferrandis et al. (2008) estudiaron la densidad de perdigones en una finca de caza menor del centro de España dedicada principalmente a la caza "en ojeo" de la perdiz roja (Alectoris rufa). La densidad reportada fue de 7.4 perdigones $/ \mathrm{m}^{2}$ a $1 \mathrm{~cm}$ de profundidad del suelo. Este dato es posiblemente bajo en comparación con otras fincas de caza intensiva de perdiz porque la frecuencia de ojeos por temporada en la zona de estudio no era de más de dos y el número de cazadores entre 6 y 16 por línea de tiro. Las densidades pueden ser mayores en otras fincas de caza intensiva en las que son liberadas perdices criadas en granjas y en donde los ojeos se pueden llevar a cabo durante toda la temporada de caza. En cuanto a la ingestión de perdigones de plomo, Soler-Rodríguez et al. (2004) examinaron la molleja de siete perdices rojas cazadas y una de ellas había ingerido 14 perdigones y presentaba $35.6 \mu \mathrm{g} / \mathrm{g}$ de plomo en peso húmedo del hígado. Más recientemente, Ferrandis et al. (2008) examinaron 76 perdices rojas cazadas en la misma finca donde se estudió la densidad de perdigones de plomo y la prevalencia de ingestión de perdigones fue del $3.9 \%$.

\section{Intoxicación por plomo en rapaces}

La intoxicación por ingestión de munición de plomo ha sido descrita en nueve especies de aves de presa diurnas y tres especies de rapaces nocturnas de España. La causa de la intoxicación es el consumo de presas muertas o heridas que presentan munición de plomo en su cuerpo. En el caso de las aves de caza, es sabido que una proporción de las aves vivas son portadoras de munición de plomo. Así por ejemplo, el $44.4 \%$ de los gansos trampeados vivos en Doñana presentaban perdigones de plomo incrustados en su cuerpo y este porcentaje llegaba a ser del $65.3 \%$ en los que fueron encontrados muertos (Mateo et al. 2007a). Se estima que el $15 \%$ de los gansos y el $19 \%$ de los patos muertos por cazadores no se recuperan, por lo que pueden ser consumidos por animales carroñeros con el consiguiente riesgo de ingestión de perdigones de plomo (USFWS 1975). Un ejemplo de esto lo dan Chapman y Buck (1893) al describir cómo un aguilucho lagunero (Circus aeruginosus) en Doñana era más rápido que ellos al cobrar las piezas que iban abatiendo en la marisma. En el caso de la caza en medio terrestre, también quedan al abasto de las rapaces presas como el conejo y la perdiz heridas o muertas. La presencia de perdigones de plomo incrustados en perdiz roja cazada fue del $87.5 \%$, con un número de perdigones incrustados entre 1 y 22 y una media de 4.19 perdigones/perdiz (Mateo et al. 2011). 
Una buena forma de evaluar el grado de exposición a la munición de plomo en las aves rapaces de forma no invasiva es mediante el examen por rayos $X$ de las egagrópilas (Fig. 3). El análisis de los restos presentes en estas egagrópilas permite además relacionar un tipo de presa con la presencia de munición (Mateo et al. 1999, 2001a). Otro tipo de muestreo no invasivo es el análisis de plumas de las rapaces (Roscales et al. 2009); sin embargo, este muestreo requiere de una interpretación adecuada, ya que el grado de contaminación externa de este metal puede ser considerable. Por este motivo, se ha visto que puede resultar más útil analizar el raquis que las barbas de la pluma para estudiar la exposición a la munición de plomo (Cardiel et al. 2011). En águila imperial ibérica (Aquila adalberti) se ha podido ver que los niveles elevados de plomo en plumas secundarias se presentan normalmente en aves con niveles elevados de plomo en huesos, y que algunas aves con niveles elevados de plomo en pluma y bajos en huesos podrían haber estado expuestas al plomo de la munición cuando la pluma ha estado en crecimiento (Rodriguez-Ramos et al. 2011).

El número de casos de plumbismo diagnosticados y publicados no es muy elevado, pero diversos estudios que han llevado a cabo análisis de tejidos de aves ingresadas en centros de recuperación de fauna silvestre o que han analizado sangre de rapaces capturadas vivas indican que los casos de plumbismo pueden ser relativamente frecuentes en aves rapaces en España. En águila real (Aquila chrysaetos) han sido detectados seis casos de intoxicación en España (Cerradelo et al. 1992, Mateo 2009). También se han detectado casos en águila imperial ibérica, una especie considerada vulnerable (VU) y que se consideraba hasta hace poco como en peligro (EN) (BirdLife 2004). La población de Doñana, que se alimenta durante el otoño-invierno de aves acuáticas malheridas o muertas, presentaba perdigones de plomo en el $14.7 \%$ de las egagrópilas analizadas durante estas estaciones en el periodo entre 1991-96 (Mateo et al. 2001a). Una menor incidencia (2.8\%) se observó en egagrópilas obtenidas a lo largo de todo el año entre 1997-2002 en Doñana (Mateo et al. 2007a) y en otro estudio anterior llevado a cabo con egagrópilas recogidas en diferentes zonas de España (0.52 \%, González e Hiraldo 1988). En CastillaLa Mancha la presencia de perdigones en egagrópilas fue del 5.5 \% durante el periodo de 1994-2004 (Castaño López 2005), observándose en esta zona un $3.6 \%$ de águilas imperiales con niveles de plomo en hueso $>20$ Mg/g p.s. (Rodríguez-Ramos et al. 2011). De hecho, en el centro de España se describió un caso de intoxicación por plomo en esta especie (Hernández 1995). Además, cuatro ejemplares, dos de ellos de Doñana, mostraron niveles de plomo en los huesos $>50 \mu \mathrm{g} / \mathrm{g}$ p.s. (Pain et al. 2005). La ingestión de perdigones de plomo en el águila imperial ibérica en Doñana puede variar entre años dependiendo de la presión de caza ejercida sobre los ánsares comunes, lo que a su vez varía en función del nivel de agua en las áreas protegidas (Mateo et al. 2007a). Una especie típica de humedales, como es el aguilucho lagunero (Circus aeruginosus), también presenta un elevado grado de exposición al perdigón de plomo. En España, la presencia de perdigones de plomo en egagrópilas de aguilucho lagunero fue de $10.7 \%$ (Mateo et al. 1999) en el Delta del Ebro y 1.8-4.3 \% en Doñana (González 1991, Mateo et al. 2007a). Las elevadas tasas de ingestión de perdigones en el Delta del Ebro se reflejan después en altos niveles de plomo en sangre $(>30 \mu \mathrm{g} / \mathrm{dl}$ ) en el $40 \%$ de las aves capturadas vivas (Mateo et al. 1999). En milano real (Milvus milvus) se ha detectado la presencia de perdigones de plomo en el $5.5 \%$ de las egagrópilas regurgitadas en Doñana al final de la temporada de caza (Mateo et al. 2001a) y un porcentaje más bajo $(1.8 \%)$ en las obtenidas a lo largo de todo el año (Mateo et al. 2007a). En hábitats terrestres del centro de España este porcentaje fue del $1.05 \%$ (García y Viñuela 1999). Esta exposición al plomo también se manifiesta en los análisis realizados en huesos de esta especie, ya que un milano real encontrado muerto en el centro de España presentó $>20 \mu \mathrm{g} / \mathrm{g}$ p.s. y otros tres tuvieron valores entre 10 y 20 mg/g (Mateo et al. 2003b).

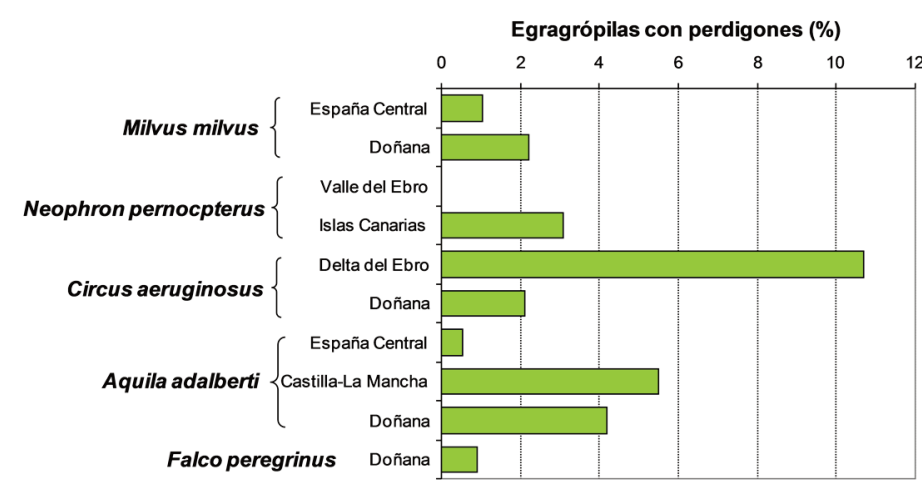

Figura 3. Presencia de perdigones de plomo en egagrópilas de aves rapaces en España.

La intoxicación por plomo se ha descrito en las cuatro especies de buitres que viven en España: buitre leonado (Gyps fulvus) (Mateo et al. 1997c), buitre negro (Aegypius monachus) (Hernández y Margalida 2008), quebrantahuesos (Gypaetus barbatus) (Hernández y Margalida 2009, Fundación Gypaetus 2010) y alimoche (Neophron percnopterus) (Rodríguez-Ramos et al. 2008). En la Sierra de Cazorla, 21 (91\%) de 23 buitres leonados capturados vivos tenían $>20 \mu \mathrm{g} / \mathrm{dl}$ de plomo en sangre y dos de ellos tuvieron $>150 \mu \mathrm{g} / \mathrm{dl}$ (García-Fernández et al. 2005). Niveles altos de plomo en sangre $(>20 \mu \mathrm{g} / \mathrm{dl})$ habían sido también observados previamente en otros seis buitres leonados en Murcia (García-Fernández et al. 1995). El alimoche en las Islas Canarias presenta una mayor vulnerabilidad a la intoxicación por plomo por el hecho de ser sedentario, a diferencia de las aves continentales que migran a África en invierno. La presencia de perdigones de plomo se detectó en el 5.3 $\%$ de las egagrópilas de esta población canaria recogidas en enero y en el $1.3 \%$ de las de noviembre. Por otra parte, cinco $(21.7 \%)$ de 23 aves tenían $>20 \mu \mathrm{g} / \mathrm{dl}$ de plomo en sangre, y una tenía $>50$ $\mu \mathrm{g} / \mathrm{dl}$ (Donazar et al. 2002). Gangoso et al. (2009) encontraron niveles elevados de plomo en sangre $(>20 \mu \mathrm{g} / \mathrm{dl})$ en $10(7.3 \%)$ alimoches de las Islas Canarias ( $n=137$ pollos y adultos), y una de esas aves mostró una concentración de $178 \mu \mathrm{g} / \mathrm{dl}$. Además, analizaron los huesos de 28 alimoches hallados muertos en las Islas Canarias, uno de los cuales resultó tener $>20 \mu \mathrm{g} / \mathrm{g}$ p.s.

Otros estudios realizados a partir de muestras de aves rapaces ingresadas en centros de recuperación han permitido detectar niveles elevados de plomo en ratonero común (Buteo buteo) (PérezLópez et al. 2008), búho real (Bubo bubo) (García-Fernández et al. 1997; Mateo et al. 2003b), búho chico (Asio otus) (Brinzal 1996) y lechuza común (Tyto alba) (González et al. 1983).

\section{Implicaciones del uso de munición de plomo para la salud pública en España}

La munición de plomo representa un riesgo para la salud pública por dos motivos. En primer lugar, las aves que han ingerido perdigones de plomo presentan unos niveles de plomo en sus tejidos, especialmente en hígado (Guitart et al. 2002) pero también en músculo (Mateo 2011), que supera en la mayoría de los casos los niveles máximos de residuos establecidos por la Unión Europea para el plomo en vísceras $(0.5 \mu \mathrm{g} / \mathrm{g}$ peso fresco, p.f.) y carne $(0.1$ $\mu \mathrm{g} / \mathrm{g}$ p.f.) de animales de granja destinados al consumo humano. En segundo lugar, la munición de plomo presente en la carne de caza puede liberar cantidades de plomo significativas durante la cocción, especialmente si ha sido cocinada con recetas que incluyen vinagre, como es el caso del escabeche (Mateo et al. 2007b). Este plomo liberado durante la cocción con vinagre presenta además una mayor biodisponibilidad (es más fácilmente absorbido) que en la carne cruda o cocinada con vino, por lo que el riesgo para el consumidor también es mayor (Mateo et al. 2011). La carne de caza mayor también puede verse contaminada por la presencia de fragmentos de balas que no son retiradas durante la preparación 
de la canal. Además, esta contaminación por la munición en la caza mayor se suma a la originada por la actividad minera que resulta en elevadas concentraciones de plomo en suelos y plantas del entorno de los lugares de extracción, contribuyendo a la acumulación del metal en ungulados y otros herbívoros (Sevillano-Morales et al. 2011, Taggart et al. 2011). Recientemente, la Agencia Española de Seguridad Alimentaria (AESAN) ha dado unas recomendaciones en cuanto al consumo de carne de caza abatida con plomo, entre las que se incluyen reducir su consumo a una ración por semana (150 g) y en el caso de niños de menos de 6 años y mujeres embarazadas o que planeen quedarse embarazadas, que se abstengan de consumirla por los efectos que tiene el plomo sobre el desarrollo del sistema nervioso central (AESAN 2012).

\section{Medidas para reducir el riesgo}

Las medidas para reducir la incidencia de plumbismo en aves y a su vez proteger a los consumidores de carne de caza pasa por utilizar municiones alternativas que no contengan plomo. Con medidas de este tipo se frena la deposición de más perdigones de plomo en los humedales y cotos de caza terrestre intensiva, se elimina el riesgo de contaminación de la carne de caza por la presencia de munición en la pieza cobrada y al mismo tiempo la posibilidad de intoxicación en las aves rapaces. Quedaría, no obstante, la contaminación residual producida por los perdigones acumulados en el medio, los cuales seguirán disponibles para las aves durante un tiempo variable en función de la deposición de nuevo sedimento en humedales, el laboreo del suelo en zonas agrícolas o las condiciones físico-químicas del suelo que faciliten su disgregación. La eliminación física de los perdigones de plomo acumulados en el suelo es prácticamente inviable en medios acuáticos, y tan solo se ha llevado a cabo con éxito en sustratos más fáciles de trabajar, como por ejemplo el Cerro de los Ánsares en Doñana (Mateo et al. 2007a). Otra posible medida para reducir la ingestión de perdigones en las aves acuáticas es el aporte de grit en las zonas húmedas en que este material es escaso, aunque los resultados obtenidos experimentalmente indican que tan solo sería efectiva en las zonas de alimentación (Mateo y Guitart 2000, Martínez-Haro et al. 2009, 2011a).

La solución realmente efectiva a largo plazo es la sustitución de la munición de plomo por otra de materiales no tóxicos como acero o cobre. El uso de plomo está restringido, al menos para la caza de aves acuáticas o sobre humedales, en 15 países de la Unión Europa (Thomas y Guitart 2010), a los que se añade además Noruega (Mateo 2009). De estos países europeos, solo cinco (Alemania, Bélgica, Dinamarca, Holanda y Suecia) han extendido total o parcialmente la prohibición del perdigón de plomo a la caza terrestre (Mateo 2009). España prohibió el uso y tenencia de munición de plomo en las zonas Ramsar y otros humedales protegidos en 2001 (Real Decreto 581/2001) y se amplió en 2007 para todos los humedales de la red Natura 2000 (Ley 42/2007) sin que ello haya afectado a las bolsas de caza en zonas como el Delta del Ebro (Fig. 4), donde el cumplimiento de la prohibición en las zonas de caza gestionadas por la Generalitat de Catalunya es prácticamente total (Mateo 2011). En cuanto a la caza en medio terrestre, tan solo se ha empezado en Cataluña a incentivar el uso de balas de cobre en caza mayor en rececho (J. Ruiz-OImo, com. pers.). También en Cazorla, con el fin de proteger al quebrantahuesos, se han realizado experiencias para que las balas de cobre sean utilizadas en las monterías (Fundación Gypaetus 2010).

A pesar del papel de la UE en la regulación de las sustancias químicas, entre las que estaría el plomo (Thomas y Guitart 2005), la Comisión Europea (CE), responsable del diseño, implementación y gestión de la política y la legislación de la UE, no ha tomado ninguna acción sobre la munición de plomo. Las convenciones internacionales que promueven la regulación del uso del plomo para la caza han sido revisadas por Thomas y Guitart $(2005,2010)$ y Mateo (2009). El Acuerdo sobre la Conservación de las Aves Acuáticas Migratorias de África y Eurasia (AEWA), dependiente de la Convención sobre la Conservación de las Especies Migratorias de Ani-

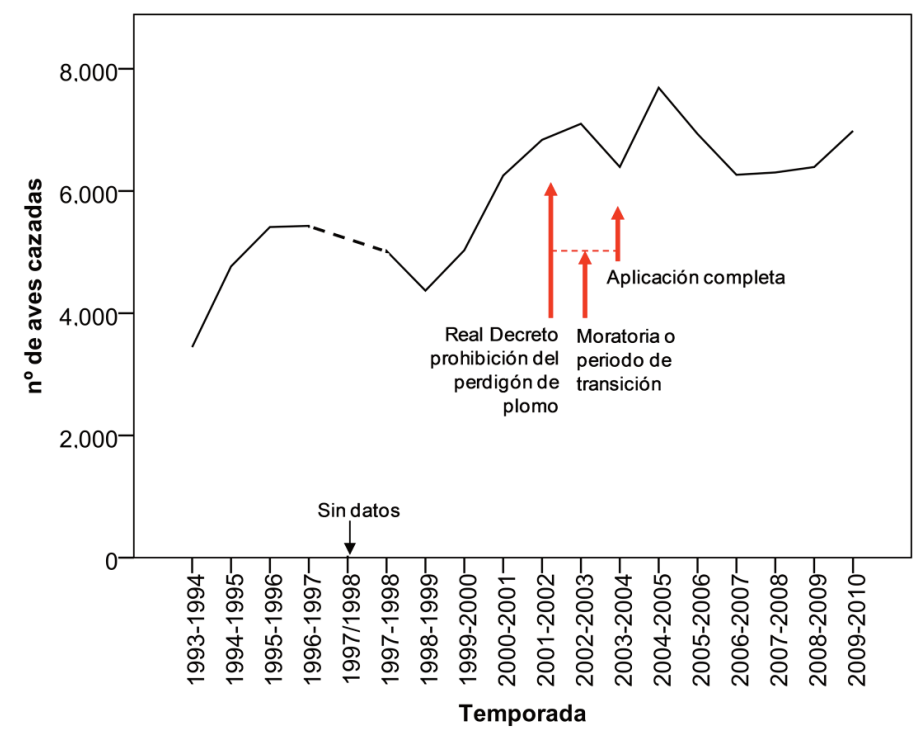

Figura 4. Evolución del número de aves abatidas en tres zonas de caza de gestión pública (Encanyissada, Isla de Buda y Garxal) del Delta del Ebro en relación a la prohibición del uso de perdigón de plomo.

males Silvestres (CMS, Convención de Bonn), ha sido el más explícito acerca de la prohibición de perdigones de plomo al decir que "las partes se esforzarán por eliminar el uso de plomo para la caza en los humedales para el año 2000". En una reciente reunión de las partes firmantes se dio un paso más al incluir en las resoluciones que "para 2017 el uso el uso de perdigones de plomo para cazar en humedales debe haber sido prohibido por los países firmantes y que (1) deberán evaluar la efectividad de las medidas nacionales adoptadas para prohibir el perdigón de plomo e implementar alternativas no tóxicas en los humedales, (2) deberán colaborar con todas las partes interesadas, cazadores, entre otros, y la industria manufacturera, para entender y abordar los obstáculos a la aplicación, y establecer y aplicar estrategias conjuntas de comunicación y (3) previa solicitud, la Secretaría y el Comité Técnico de la AEWA prestará apoyo para la realización de dicha colaboración y evaluación" (AEWA 2012).

\section{Agradecimientos}

Esta revisión se enmarca dentro del proyecto financiado por el MICINN con referencia CGL2010-17030/BOS. NV disfruta de una beca FPI asociada a dicho proyecto. MEOS disfruta de un contrato del programa Juan de la Cierva.

\section{Referencias}

AESAN 2012. Informe del Comité Científico de la Agencia Española de Seguridad Alimentaria y Nutrición (AESAN) sobre el riesgo asociado a la presencia de plomo en carne de caza silvestre en España. Revista del Comité Científico 15:131-159

AEWA 2012. Resolution 5.231. AEWA's contribution to delivering the Aichi 2020 biodiversity targets. 5th Session of the Meeting of the Parties 1418 May 2012, La Rochelle, France. www.unep-aewa.org/meetings/en/mop/mop5 docs/mop5 final res.htm. Consultado el 3 de febrero de 2013.

BirdLife International 2004. Birds in Europe: Population Estimates, Trends and Conservation Status. BirdLife International, Wageningen, The Netherlands.

BirdLife International 2012. Oxyura leucocephala. In: IUCN 2012. IUCN Red List of Threatened Species. Version 2012.2. www.iucnredlist.org. Descargado el 3 de febrero de 2013.

Bonet, A., Olivares, C., Picó, M. L., Sales. S. 1995. L'acumulació de perdigons de plom al Parc Natural del Fondó d'Elx (Alacant): distribució espacial i propostes d'actuació. Butlletí de la Instució Catalana d'Història Natural 63:149-166.

Bonet, A., Terrones, B., Peña. J. 2004. El Hondo, a la cabeza en cantidad de perdigones de plomo depositados. Quercus 221:66-67. 
Brinzal 1996. S.O.S. Venenos: Búho chico. Quercus 124:45.

Castaño Lopez, J.P. 2005. El Águila Imperial Ibérica en Castilla La Mancha. Status, Ecología y Conservación. Graphitis Impresores, Madrid.

Cardiel, I.E., Taggart, M.A., Mateo, R. 2011. Use of the ratio Pb-Al to discriminate between internal and external deposition of $\mathrm{Pb}$ in feathers. Ecotoxicology and Environmental Safety 74: 911-917.

Cerradelo, S., Muñoz, E., To-Figueras, J., Mateo, R., Guitart, R. 1992. Intoxicación por ingestión de perdigones de plomo en dos Águilas Reales. Doñana, Acta Vertebrata 19:122-127.

Chapman, A., Buck, W.J. 1893. Wild Spain. Gurney and Jackson, London, UK.

Donázar, J.A., Palacios, C.J., Gangoso, L., Ceballos, O., González, M.J., Hiraldo, F. 2002. Conservation status and limiting factors in the endangered population of Egyptian Vulture (Neophron percnopterus) in the Canary Islands. Biological Conservation 107:89-97.

Ferrandis, P., Mateo, R., López-Serrano, F.R., Martínez-Haro, M., MartínezDuro, E. 2008. Lead-shot exposure in red-legged partridge (Alectoris rufa) on a driven shooting estate. Environmental Science and Technology 42:6271-6277.

Figuerola, J., Mateo, R., Green, A.J., Mondain-Monval, J.-Y., Lefranc, H., Mentaberre, G. 2005. Interspecific and spatial variability in the ingestion of grit and lead shot by waterfowl. Environmental Conservation 32:226-234.

Fundación Gypaetus 2010. Memoria de Actividades 2010. www.gypaetus.org. Descargado el 3 de febrero de 2013.

Gangoso, L., Álvarez-Lloret, P., Rodríguez-Navarro, A., Mateo, R., Hiraldo F., Donázar, J. A. 2009. Long-term effects of lead poisoning on bone mineralization in Egyptian Vulture Neophron percnopterus. Environmental Pollution 157: 569-574.

García-Fernández, A.J., Sánchez-García, J.A., Jiménez-Montalbán, P., Lunas A. 1995. Lead and cadmium in wild birds in southeastern Spain Environmental Toxicology and Chemistry 14:2049-2058.

García-Fernández, A.J., Motas-Guzmán, M., Navas, I., María-Mojica, P., Luna, A., Sánchez-García, J.A. 1997. Environmental exposure and distribution of lead in four species of raptors in southeastern Spain Archives of Environmental Contamination and Toxicology 33:76-82.

García-Fernández, A.J., Martínez-López, E., Romero, D., María-Mojica, P., Godino, A., Jiménez P. 2005. High levels of blood lead in Griffon Vultures (Gyps fulvus) from Cazorla Natural Park (southern Spain). Environmental Toxicology 20:459-463.

García, J.T., Viñuela, J. 1999. El plumbismo: una primera aproximación en el caso del Milano Real. En: Viñuela, J., Martí, R., Ruiz, A. (eds.), El Milano Real en España. pp. 213-220. Sociedad Española de Ornitología/BirdLife, Madrid, España.

González, J.L. 1991. El Aguilucho Lagunero (Circus aeruginosus) en España. ICONA-CSIC, Madrid, España.

González, L.M., Hiraldo, F. 1988. Organochlorine and heavy metals contamination in the eggs of the Spanish Imperial Eagle (Aquila adalberti) and accompanying changes in eggshell morphology and chemistry. Environmental Pollution 51:241-258.

González, M.J., Rico, M.C., Fernández-Aceytuno, M.C., Hernández, L.M., Baluja, G. 1983. Contaminación xenobiótica del Parque Nacional de Doñana. II. Residuos de insecticidas organoclorados, bifenilos policlorados (PCBs) y metales pesados en Falconiformes y Strigiformes. Doñana, Acta Vertebrata 10:177-189.

Guitart, R., To-Figueras, J., Mateo, R., Bertolero, A., Cerradelo, S., MartínezVilalta, A. 1994a. Lead poisoning in waterfowl from the Ebro delta Spain: Calculation of lead exposure thresholds for Mallards. Archives of Environmental Contamination and Toxicology 27:289-293.

Guitart, R., Torra, M., Cerradelo, S., Puig-Casado, P., Mateo, R., ToFigueras, J. 1994b. Pb, Cd, As, and Se concentrations in livers of dead wild birds from the Ebro delta, Spain. Bulletin of Environmental Contamination and Toxicology 52:523-529.

Guitart, R., Mañosa, S., Thomas, V.G., Mateo, R. 1999. Perdigones y pesos de plomo: ecotoxicología y efectos para la fauna. Revista de Toxicología 16:3-11.

Guitart, R., Serratosa, J., Thomas, V.G. 2002. Lead-poisoned wildfowl in Spain: a significant threat for human consumers. International Journal of Environmental Health Research 12:301-309.

Hernández, M. 1995. Lead poisoning in a free-ranging Imperial Eagle. Supplement to the Journal of Wildlife Diseases 31 (3), Newsletter.

Hernández, M., Margalida, A. 2008. Pesticide abuse in Europe: effects on the Cinereous Vulture (Aegypius monachus) population in Spain. Ecotoxicology 17:264-272.
Hernández, M., Margalida, A. 2009. Assessing the risk of lead exposure for the conservation of the endangered Pyrenean bearded vulture (Gypaetus barbatus) population. Environmental Research 109:837-842.

Jørgensen, S.S., Willems, M. 1987. The fate of lead in soils: the transformation of lead pellets in shooting-range soils. Ambio 16:11-15.

Llorente, G.A. 1984. Contribución al conocimiento de la biología y la ecología de cuatro especies de anátidas en el delta del Ebro. Tesis Doctoral, Universidad de Barcelona, Barcelona, España.

Martínez-Haro, M., Taggart, M.A., Green, A.J., Mateo, R. 2009. An avian digestive tract simulation to study the effect of grit geochemistry and food on $\mathrm{Pb}$ shot bioaccessibility. Environmental Science and Technology 43:9480-9486.

Martínez-Haro, M., Taggart, M.A., Mateo, R. 2010. Lead shot or soil ingestion? $\mathrm{Pb}-\mathrm{Al}$ relationships in waterfowl feces discriminate between sources of $\mathrm{Pb}$ exposure. Environmental Pollution 158:2485-2489.

Martínez-Haro, M., Green, A.J., Acevedo, P., Mateo, R. 2011a. Use of grit supplements by waterbirds: an experimental assessment of strategies to reduce lead poisoning. European Journal of Wildlife Research 57:475-484.

Martínez-Haro, M., Green, A.J., Mateo, R. 2011b. Effects of Pb exposure on oxidative stress biomarkers and plasma biochemistry in waterbirds in the field. Environmental Research 111: 530-538

Martinez-Haro, M., Taggart, M.A., Rodríguez Martín-Doimeadios, R., Green, A.J., Mateo, R. 2011c. Identifying sources of Pb exposure in waterbirds and effects on porphyrin metabolism using non-invasive fecal sampling. Environmental Science and Technology 45: 6153-6159

Martinez-Haro, M., Taggart, M., Lefranc, H., Martín-Doimeadiós, R.C., Green, A.J., Mateo, R. 2013. Monitoring of Pb exposure in waterfowl ten years after a mine spill through the use of noninvasive sampling. PLoS ONE 8:e57295.

Mateo, R. 1998. La intoxicación por ingestión de perdigones de plomo en aves silvestres: aspectos epidemiológicos y propuestas para su prevención en España. Tesis Doctoral, Universitat Autònoma de Barcelona, Bellaterra, España.

Mateo, R. 2009. Lead poisoning in wild birds in Europe and the regulations adopted by different countries. En: Watson R.T., Fuller, M., Pokras, M. Hunt, W.G. (eds.), Ingestion of Lead from Spent Ammunition: Implications for Wildlife and Humans, pp. 71-98. The Peregrine Fund, Boise, Idaho, USA.

Mateo, R. 2011. No contaminem l'ambient i el menjar amb el plom dels perdigons! Soldó 37: 25-26

Mateo, R., Guitart, R. 2000. The effects of grit supplementation and feed type on steel-shot ingestion in Mallards. Preventive Veterinary Medicine 44:221-229.

Mateo, R., Dolz, J.C., Aguilar-Serrano, J.M., Belliure, J., Guitart, R. 1997a. An outbreak of lead poisoning in Greater Flamingos Phoenicopterus ruber roseus in Spain. Journal of Wildlife Diseases 33:131-134.

Mateo, R., Guitart, R., Martínez-Vilalta, A. 1997b. Lead shot pellets in the Ebro delta, Spain: Densities in sediments and prevalence of exposure in waterfowl. Environmental Pollution 96:335-341.

Mateo, R., Molina, R., Grífols, J., Guitart, R. 1997c. Lead poisoning in a free ranging Griffon Vulture (Gyps fulvus). Veterinary Record 140:47-48

Mateo, R., Belliure, J., Dolz, J.C., Aguilar-Serrano, J.M., Guitart, R. 1998a. High prevalences of lead poisoning in wintering waterfowl in Spain. Archives of Environmental Contamination and Toxicology 35:342-347.

Mateo, R., Grífols, J., Molina, R., Martínez, F., Guitart, R. 1998b. Intoxicación en aves por ingestión de objetos de plomo. Consulta de Difusión Veterinaria 6:2014-2015.

Mateo, R., Estrada, J., Paquet, J.-Y., Riera, X., Dominguez, L., Guitart, R., Martínez-Vilalta A. 1999. Lead shot ingestion by Marsh Harriers Circus aeruginosus from the Ebro delta, Spain. Environmental Pollution 104:435-440.

Mateo, R., Bonet A., Dolz J.C., Guitart R. 2000a. Lead shot densities in a site of grit ingestion for Greylag Geese Anser anser in Doñana (Spain). Ecotoxicology and Environmental Restoration 3:76-80.

Mateo, R., Guitart, R., Green, A.J. 2000b. Determinants of lead shot, rice, and grit ingestion in ducks and coots. Journal of Wildlife Management 64:939-947.

Mateo, R., Cadenas, R., Máñez, M., Guitart, R. 2001a. Lead shot ingestion in two raptor species from Doñana, Spain. Ecotoxicology and Environmental Safety 48:6-10. 
Mateo, R., Green, A.J., Jeske, C.V., Urios, V., Gerique, C. 2001b. Lead poisoning in the globally threatened Marbled Teal and White-headed Duck in Spain. Environmental Toxicology and Chemistry 20:2860-2868.

Mateo, R., Beyer, W.N., Spann, J.W., Hoffman, D.J., Ramis, A. 2003a. Relationship between oxidative stress, pathology and behavioural signs of lead poisoning in mallards. Journal of Toxicology and Environmental Health Part A 66:1371-1389.

Mateo, R., Taggart, M., Meharg A.A. 2003b. Lead and arsenic in bones of birds of prey from Spain. Environmental Pollution 126:107-114.

Mateo, R., Castells, G., Green, A.J., Godoy, C., Cristòfol, C. 2004. Determination of porphyrins and biliverdin in bile and excreta of birds by a single liquid chromatography-ultraviolet detection analysis. Journal of Chromatography B 810:305-311.

Mateo, R., Taggart, M.A., Green, A.J., Cristòfol, C., Ramis, A., Lefranc, H., Figuerola, J., Meharg, A.A. 2006. Altered porphyrin excretion and histopathology of Greylag Geese (Anser anser) exposed to soil contaminated with lead and arsenic in the Guadalquivir Marshes, SW Spain. Environmental Toxicology and Chemistry 25: 203-212.

Mateo, R., Green, A.J., Lefranc, H., Baos, R., Figuerola J. 2007a. Lead poisoning in wild birds from southern Spain: A comparative study of wetland areas and species affected, and trends over time. Ecotoxicology and Environmental Safety 66:119-126.

Mateo, R., Rodríguez-de la Cruz, M., Vidal, D., Reglero, M., Camarero, P. 2007b. Transfer of lead from shot pellets to game meat during cooking. Science of the Total Environment 372:480-485.

Mateo, R., Baos, A.R., Vidal, D., Camarero, P.R., Martínez-Haro, M., Taggart, M.A. 2011. Bioaccessibility of $\mathrm{Pb}$ from ammunition in game meat is affected by cooking treatments. PLoS ONE 6:e15892.

Pain, D.J., Meharg A.A., Ferrer, M., Taggart, M.A., Penteriani, V. 2005. Lead concentrations in bones and feathers of the globally threatened Spanish Imperial Eagle. Biological Conservation 121:603-610.

Pérez-López, M., Hermoso de Mendoza, M., López Beceiro, A., Soler Rodríguez, F. 2008. Heavy metal (Cd, Pb, Zn) and metalloid (As) content in raptor species from Galicia (NW Spain). Ecotoxicology and Environmental Safety 70:154-162.

Ramo, C., Sánchez, C., Hernández Saint-Aubin, L. 1992. Lead poisoning of Greater Flamingos Phoenicopterus ruber. Wildfowl 43:220-222.

Rodriguez, R., Hiraldo, F. 1975. Régimen alimenticio del Calamón Porphyrio porphyrio en las Marismas del Guadalquivir. Doñana Acta Vertebrata 2:201-213

Rodríguez, J.J., Oliveira, P.A., Hidalgo, L.E., Ginja, M.M., Silvestre, A.M., Ordoñez, C., Serantes, A.E., Gonzalo-Orden, J.M., Orden, M.A. 2010. Lead toxicity in captive and wild Mallards (Anas platyrhynchos) in Spain. Journal of Wildlife Diseases 46:854-63.

Rodríguez-Ramos, J., Gutierrez, V., Höfle, U., Mateo, R., Monsalve, L., Crespo, E., Blanco, J. M. 2008. Lead in Griffon and Cinereous Vultures in Central Spain: Correlations between clinical signs and blood lead lev- els. En: Watson R.T., Fuller, M., Pokras, M. Hunt, W.G. (eds.), Ingestion of Lead from Spent Ammunition: Implications for Wildlife and Humans, pp. 235-236. The Peregrine Fund, Boise, Idaho, USA.

Rodríguez-Ramos, J., Hofle, U., Mateo, R., Nicolas, O., Abbott, R., Acevedo, P., Blanco, J.M. 2011. Assessment of lead exposure in Spanish imperial eagle (Aquila adalberti) from spent ammunition in central Spain. Ecotoxicology 20:670-681

Roscales, J.L., Sáez, M., Blázquez, E., Ferrer, M., Gil, J.A., Mateo, R., Jiménez, B. 2009. Evaluación no destructiva de la exposición a plomo en rapaces amenazadas en parques nacionales: el Águila Imperial Ibérica (Aquila adalberti) y el Quebrantahuesos (Gypaetus barbatus). En: Ramírez, L., Asensio, B. (eds.), Proyectos de Investigación en Parques Nacionales: 2005-2008, pp. 215-228, Organismo Autónomo Parques Nacionales, Madrid, España.

Sevillano Morales, J., Moreno Rojas, R., Pérez-Rodríguez, F., Arenas Casas, A., Amaro López M. 2011. Risk assessment of the lead intake by consumption of red deer and wild boar meat in Southern Spain. Food Additives and Contaminants Part A 28:1021-1033.

Soler-Rodríguez, F., Oropesa-Jiménez, A.L., García-Cambero, J.P., PérezLópez, M. 2004. Lead exposition by gunshot ingestion in Red-legged Partridge (Alectoris rufa). Veterinary and Human Toxicology 46:133-134.

Svanberg, F., Mateo, R., Hillström, L., Green, A.J., Taggart, M.A., Raab, A., Meharg A.A. 2006. Lead isotopes and lead shot ingestion in the globally threatened Marbled Teal (Marmaronetta angustirostris) and Whiteheaded Duck (Oxyura leucocephala). Science of the Total Environment 370:416-424.

Taggart, M.A., Green, A.J, Mateo, R., Svanberg, F., Hillström, L., Meharg, A.A. 2009. Metal levels in the bones and livers of globally threatened marbled teal and white-headed duck from El Hondo, Spain. Ecotoxicology and Environmental Safety 72: 1-9.

Taggart, M.A., Reglero, M.M., Camarero, P.R., Mateo, R. 2011. Should legislation regarding maximum heavy metal levels in human food also cover large game meat? Environment International 37:18-25.

Thomas, V.G., Guitart, R. 2005. Role of international conventions in promoting avian conservation through reduced lead toxicosis: Progression towards a non-toxic agenda. Bird Conservation International 15:147-160.

Thomas, V.G., Guitart. R. 2010. Limitations of European Union policy and law for regulating use of lead shot and sinkers: Comparisons with North American regulation. Environmental Policy and Governance 20:57-72.

Torres-Esquivias, J.A. 2008. La malvasía cabeciblanca. En: Ballesteros, G., Cabrera, M., Echevarrías, J.L., Lorenzo, J.A., Raya, C., Torres-Esquivias, J.A., Viedma, C. (eds.), pp. 54-69. Tarro Canelo, Cerceta Pardilla, Porrón Pardo, Malvasía Cabeciblanca y Focha Moruna en España. Población en 2007 y Método de Censo. SEO/BirdLife, Madrid, España.

USFWS 1975. Issuance of Annual Regulations Permitting the Sport Hunting of Migratory Birds. Final Environmental Statement. U.S. Fish and Wildlife Service, Washington, D.C., USA.. 